\title{
Correction to: Multi-level, multi-scale habitat selection by a wide-ranging, federally threatened snake
}

\author{
Javan M. Bauder • David R. Breininger - M. Rebecca Bolt • Michael L. Legare • \\ Christopher L. Jenkins • Betsie B. Rothermel $\cdot$ Kevin McGarigal
}

Published online: 7 August 2018

(C) Springer Nature B.V. 2018

Correction to: Landscape Ecol (2018) 33:743-763

https://doi.org/10.1007/s10980-018-0631-2

In the original publication of this article, the authors discovered that they mistakenly used urban instead of urban edge rasters for two of their four study areas. As a result, the urban edge covariates in their analyses were a mixture of urban and urban edge making the inferences regarding urban edge in the original publication incorrect. After correctly measuring urban edge, the correct characteristic scale for the Level II analysis was $1100 \mathrm{~m}$ and the correct characteristic scales for the Level III analyses were $h=75,15,30$, and $165 \mathrm{~m}$ for breeding and non-breeding season

The original article can be found online

at https://doi.org/10.1007/s10980-018-0631-2.

J. M. Bauder $(\bowtie) \cdot$ K. McGarigal

Department of Environmental Conservation, University of

Massachusetts, 160 Holdsworth Way, Amherst,

MA 01003, USA

e-mail: javanvonherp@gmail.com

D. R. Breininger

NASA Ecological Programs, Integrated Mission Support Services, Mail Code IMSS-300, Kennedy Space Center, FL 32899, USA

M. R. Bolt

NASA Ecological Programs, Integrated Mission Support Services, Mail Code IMSS-200, Kennedy Space Center, FL 32899, USA females and males, respectively. The equal weights scenario was the best supported weighting scenario for urban edge. The authors excluded urban edge from the Level II analysis and the Level III analyses for breeding season females and non-breeding season males because of high collinearity $(r \geq 0.65)$ with other covariates. The authors reran their analyses and found that inferences regarding other covariates and their overall conclusions remained unchanged.

The second paragraph in the Level II subsection of the Methods should read "At Level II, undeveloped ( $h=60 \mathrm{~m}$ ), SD of spring NDVI (radius $=60 \mathrm{~m}$ ), and wetland edge ( $h=15 \mathrm{~m}$ ) received the strongest support (parameter weights $=0.87,0.65$, and 0.54 , respectively) although all had model-averaged $95 \%$ CI that overlapped zero. Post-hoc analyses including urban and roads indicated moderate to low support for

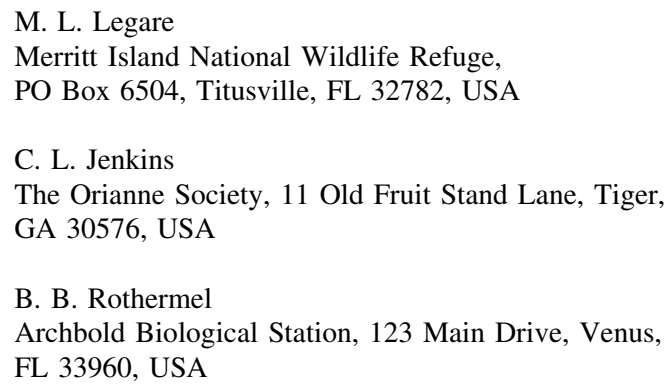


these covariates (parameter weights $=0.66$ and 0.33 , respectively, with model-averaged $95 \%$ CI overlapping zero). The predictive performance of the top model was high $(\mathrm{CCC}=0.91,0.54-0.98)$ when crossvalidating by site and $\mathrm{D}^{2}=0.11$. Model-averaged $\mathrm{CCC}$ and $\mathrm{D}^{2}$ across the $90 \%$ model set $(n=125)$ was $0.88 \quad$ (range $=0.47-0.99$ ) and 0.09 (range $=$ 0.03-0.11), respectively."

For the Level III analyses, the correct modelaveraged beta estimate, $95 \% \mathrm{CI}$, and parameter weight for urban edge for non-breeding season females in Table 5 was $0.29,-0.11$ to 0.69 , and 0.96 , respectively. The correct model-averaged $\mathrm{D}^{2}$ across the $90 \%$ model set was $0.22,0.21,0.32$, and 0.22 for breeding and non-breeding season females and males, respectively. The correct model-averaged CCC was 0.66 (0.59-0.72), 0.73 (0.60-0.82), 0.89 (0.87-0.96), and $0.78(0.77-0.88)$ for breeding and non-breeding season females and males, respectively.

The authors also mistakenly reported the median eastern indigo snake (EIS) total home range size in the first paragraph of the Predicted Surfaces section in the Methods. The median EIS total home range size was 144 ha and the radius of the circular Uniform kernel used to approximate a median EIS total home range was $677 \mathrm{~m}$. We deeply apologize for these mistakes and any inconveniences they may cause. 